\title{
Measurement-Induced Nonlocality and Geometric Discord in the Spin-Boson Model
}

\author{
Guoyou Wang, Zilong Fan, Haosheng Zeng* \\ Key Laboratory of Low-Dimensional Quantum Structures and Quantum Control of Ministry of \\ Education, Department of Physics, Hunan Normal University, Changsha, China \\ Email: "hszeng@hunnu.edu.cn
}

Received December 9, 2012; revised January 22, 2013; accepted February 1, 2013

\begin{abstract}
Dynamics of measurement-induced-nonlocality (MIN) and geometric measure of discord (GD) in the spin-boson model are studied. Analytical results show that for two large classes of initial states, MINs are equal but GDs are different. At the end of evolution, MIN and GD initially stored in the spin system transfer completely to reservoirs. The quantum beats for MIN and GD are also found, which are the results of quantum interference between two local non-Markovian dynamics via quantum correlation.
\end{abstract}

Keywords: Measurement-Induced Nonlocality; Geometric Discord; Quantum Beat

\section{Introduction}

Quantum correlation arises from noncommutativity of operators representing states, observables, and measurements [1]. Quantum entanglement, as the earliest known quantum correlation, has acquired extensive research and is found to be an useful resource in quantum communication and quantum computation [2]. However, entanglement is not the unique kind of quantum correlation. A more general concept, quantum discord [3], was found, which is regarded as the measure of all nonclassical correlations in a bipartite system, being the entanglement of a particular case of it. It was shown that there exists separable states with nonzero discord which can be used to perform quantum computation [4,5]. Quantum discord is also useful in the studies of quantum phase transition $[6,7]$ and estimation of quantum correlations in Grover search algorithm [8]. Unfortunately, evaluation of quantum discord in general requires considerable numerical minimization and analytical results that are known only for certain classes of states [9-11]. More recently, two measures out of geometric perspective in measurements, GD [12] and MIN [13], were proposed, which as the authors shown have the merit of easier evaluation. In fact, Luo and $\mathrm{Fu}$ $[14,15]$ have evaluated the GD for some typical classes of states and found the tight lower bound. The dissecting about the meaning of GD was also done [16,17].

Realistic quantum systems cannot avoid interactions with their environments, leading to the change of quantum correlation. In the last decade, the influences of en-

*Corresponding author. vironments to quantum entanglement [18] and quantum discord [19] have been investigated extensively. An interesting phenomenon, named as "entanglement sudden death" (ESD) $[20,21]$ for a pair of entangled qubits exposed to local Markovian environments was found. In contrast, quantum discord in similar conditions decays only in asymptotic time [22], which signifies that quantum discord is more robust against Markovian noise than entanglement. There are also many works involved in the evolutions of quantum entanglement and discord in the non-markovian environments [23-29]. Especially, trapping [30] and quantum beats [31] for quantum entanglement and discord for a pair of qubits in local structured environments were found.

In this paper, we investigate the dynamics of GD and MIN in a system that consists of two independent spins (qubits) coupled respectively to their local environments. Our motivation is to find the evolutional properties of GD and MIN and the difference between their evolutions and make a comparison between the evolutions of quantum entanglement or/and discord. The paper is constructed as follows. In Section 2, we introduce the original definitions of GD and MIN. In Section 3, we first introduce the interaction model, and then study the evolution of GD and MIN of different partitions for two classes of initial states. Section 4 is devoted to the study of quantum beats for GD and MIN. And the conclusion is arranged in Section 5.

\section{GD and MIN}

Let $\rho$ be a bipartite state shared by parties A and B. The GD of $\rho$ is defined as [12], 


$$
D(\rho)=\min _{\Pi^{A}}\left\|\rho-\Pi^{A}(\rho)\right\|^{2},
$$

where the min is taken over all von Neumann measurements $\Pi^{A}$ on subsystem $A$, and the Hilbert Schmidt norm defined by $\|X\|^{2}=\operatorname{tr} X^{\dagger} X$. On the other hand, the MIN is defined as [13]

$$
N(\rho)=\max _{\Pi^{A}}\left\|\rho-\Pi^{A}(\rho)\right\|^{2},
$$

here the max is taken over the von Neumann measurements which do not disturb $\rho^{A}$ locally, that is,

$$
\sum_{k} \Pi_{k}^{A} \rho^{A} \Pi_{k}^{A}=\rho^{A} .
$$

For a two-qubit system, the general state may be written as,

$$
\begin{aligned}
& \rho=\frac{1}{4}\left(\mathbf{1}^{A} \otimes \mathbf{1}^{B}+\sum_{i=1}^{3} x_{i} \sigma_{i}^{A} \otimes \mathbf{1}^{B}\right. \\
& \left.+\sum_{i=1}^{3} y_{i} \mathbf{1}^{A} \otimes \sigma_{i}^{B}+\sum_{i, j=1}^{3} t_{i j} \sigma_{i}^{A} \otimes \sigma_{j}^{B}\right) .
\end{aligned}
$$

Here $\sigma_{i}$ are the Pauli operators, $x_{i}=\operatorname{Tr} \rho\left(\sigma_{i}^{A} \otimes \mathbf{1}^{B}\right)$ and $y_{i}=\operatorname{Tr} \rho\left(\mathbf{1}^{A} \otimes \sigma_{i}^{B}\right)$ are the components of the local Bloch vectors, $t_{i j}=\operatorname{Tr} \rho\left(\sigma_{i}^{A} \otimes \sigma_{j}^{B}\right)$ are the components of the correlation tensor $T=\left[t_{i j}\right]$. For this state, we have $[12,13]$

$$
D(\rho)=\frac{1}{4}\left(\|x\|^{2}+\|T\|^{2}-k_{\max }\right)
$$

and

$$
N(\rho)= \begin{cases}\frac{1}{4}\left(\operatorname{tr} T T^{t}-\frac{1}{\|x\|^{2}} x^{t} T T^{t} x\right) & \text { if } x \neq 0, \\ \frac{1}{4}\left(\operatorname{tr} T T^{t}-\lambda_{3}\right) & \text { if } x=0 .\end{cases}
$$

where $\|x\|^{2}=\sum_{i} x_{i}^{2}$ with $\boldsymbol{x}=\left(x_{1}, x_{2}, x_{3}\right)^{t}, k_{\max }$ is the maximum eigenvalue of matrix $x x^{t}+T T^{t}$, and $\lambda_{3}$ the minimum eigenvalue of matrix $T T^{t}$.

\section{Model and Dynamics of MIN and GD}

The model we consider consists of two independent spins interacting respectively with their local boson reservoirs. The total Hamiltonian is,

$$
\begin{aligned}
H & =\sum_{i=1}^{2}\left(\frac{\omega_{0}}{2} \sigma_{i}^{2}+\sum_{k} \omega_{i, k} b_{i, k}^{\dagger} b_{i, k}\right) \\
& +\sum_{i=1}^{2} \sum_{k} g_{i, k}\left(\sigma_{i}^{-} b_{i, k}^{\dagger}+\sigma_{i}^{+} b_{i, k}\right) .
\end{aligned}
$$

Here $\sigma_{i}^{z}$ and $\sigma_{i}^{+}\left(\sigma_{i}^{-}\right)$are the Pauli and raising (low- ering) operators for the ith spin. $b_{i, k}^{\dagger}\left(b_{i, k}\right)$ is the creation (annihilation) operator of the ith mode in the ith reservoir with corresponding frequency $\omega_{i, k}$. The coupling strength between spin $i$ and its reservoir mode $k$ is denoted by $g_{i, k}$. For simplicity, we assume the two spins have equal Zeeman splitting $\omega_{0}$, and the reservoirs are initially in vacuum states and not correlated to the spins. Under these conditions, the model can be solved exactly for any initial state of the spins and any form of spectral reservoirs. In this paper, we will mainly discuss two types of reservoirs - unstructured flat reservoirs and structured Lorentzian reservoirs, and also two large types of initial states of spins - double-excitation and one-excitation states.

\subsection{Double-Excitation Dynamics of MIN and GD}

Let us first study the double-excitation case, i.e., the joint initial state for the whole system is,

$$
|\phi(0)\rangle=\left(\alpha|0\rangle_{s_{1}}|0\rangle_{s_{2}}+\beta|1\rangle_{s_{1}}|1\rangle_{s_{2}}\right)|0\rangle_{r_{1}}|0\rangle_{r_{2}},
$$

where $|\alpha|^{2}+|\beta|^{2}=1$, and the collective state $|0\rangle_{r_{i}}=\prod_{k}\left|0_{k}\right\rangle_{r_{i}}$ represents vacuum state of reservoir $r_{i}$.

Due to the conservation of excitation number under the evolution of Jaynes-Cummings model, the dynamical state of the whole system has the form,

$$
\begin{aligned}
|\phi(t)\rangle= & \beta\left[\xi_{1}(t)|1\rangle_{s_{1}}|0\rangle_{r_{1}}+\chi_{1}(t)|0\rangle_{s_{1}}|1\rangle_{r_{1}}\right] \\
& \times\left[\xi_{2}(t)|1\rangle_{s_{2}}|0\rangle_{r_{2}}+\chi_{2}(t)|0\rangle_{s_{2}}|1\rangle_{r_{2}}\right] \\
& +\alpha|0\rangle_{s_{1}}|0\rangle_{s_{2}}|0\rangle_{r_{1}}|0\rangle_{r_{2}} .
\end{aligned}
$$

Here we introduce the collective state $|1\rangle_{r_{i}}=\frac{1}{\chi_{i}(t)} \sum_{k} \lambda_{k}(t)\left|1_{k}\right\rangle_{r_{i}}$ to denote the one-excitation state of the reservoir $r_{i}$ [19,32]. The coefficients $\xi_{i}(t)$ and $\chi_{i}(t)$, which are determined by the quantum dynamical equation, satisfy

$$
\xi_{i}(t)=-\int_{0}^{t} \mathrm{~d} t_{1} f\left(t-t_{1}\right) \xi_{i}\left(t_{1}\right)
$$

and

$$
\left|\xi_{i}(t)\right|^{2}+\left|\chi_{i}(t)\right|^{2}=1 \text {. }
$$

Here the kernel function is defined by

$$
f_{i}\left(t-t_{1}\right)=\int \mathrm{d} \omega J_{i}(\omega) \mathrm{e}^{i\left(\omega_{0}-\omega\right)\left(t-t_{1}\right)},
$$

with spectral density $J_{i}(\omega)=\sum_{k}\left|g_{i, k}\right|^{2} \delta\left(\omega-\omega_{i, k}\right)$.

The reduced density matrix for the two spins reads 


$$
\rho_{s_{1} s_{2}}=\left(\begin{array}{cccc}
|\alpha|^{2}+\left|\beta \chi_{1} \chi_{2}\right|^{2} & 0 & 0 & \alpha\left(\beta \xi_{1} \xi_{2}\right)^{*} \\
0 & \left|\beta \chi_{1} \xi_{2}\right|^{2} & 0 & 0 \\
0 & 0 & \left|\beta \xi_{1} \chi_{2}\right|^{2} & 0 \\
\alpha^{*} \beta \xi_{1} \xi_{2} & 0 & 0 & \left|\beta \xi_{1} \xi_{2}\right|^{2}
\end{array}\right)
$$

From Equations (4) and (5), the analytical expressions for MIN and GD can be written as

$$
N\left(\rho_{s_{1} s_{2}}\right)=2\left|\alpha \beta \xi_{1} \xi_{2}\right|^{2},
$$

and

$$
\begin{aligned}
& D\left(\rho_{s_{1} s_{2}}\right) \\
& =\frac{1}{4}\left\{\left(1-2\left|\beta \xi_{1}\right|^{2}\right)^{2}+8\left|\alpha \beta \xi_{1} \xi_{2}\right|^{2}\right. \\
& +\left(1-2\left|\beta \chi_{1} \xi_{2}\right|^{2}-2\left|\beta \xi_{1} \chi_{2}\right|^{2}\right)^{2} \\
& -\max \left[4\left|\alpha \beta \xi_{1} \xi_{2}\right|^{2},\left(1-2\left|\beta \xi_{1}\right|^{2}\right)^{2}\right. \\
& \left.\left.+\left(1-2\left|\beta \chi_{1} \xi_{2}\right|^{2}-2\left|\beta \xi_{1} \chi_{2}\right|^{2}\right)^{2}\right]\right\} .
\end{aligned}
$$

Similarly, the MIN and GD for the reservoirs reads,

$$
\begin{gathered}
N\left(\rho_{r_{1} r_{2}}\right)=2\left|\alpha \beta \chi_{1} \chi_{2}\right|^{2}, \\
D\left(\rho_{r_{1} r_{2}}\right)=\frac{1}{4}\left\{\left(1-2\left|\beta \chi_{1}\right|^{2}\right)^{2}+8\left|\alpha \beta \chi_{1} \chi_{2}\right|^{2}\right. \\
+\left(1-2\left|\beta \xi_{1} \chi_{2}\right|^{2}-2\left|\beta \chi_{1} \xi_{2}\right|^{2}\right)^{2} \\
-\max \left[4\left|\alpha \beta \chi_{1} \chi_{2}\right|^{2},\left(1-2\left|\beta \chi_{1}\right|^{2}\right)^{2}\right. \\
\left.\left.+\left(1-2\left|\beta \xi_{1} \chi_{2}\right|^{2}-2\left|\beta \chi_{1} \xi_{2}\right|^{2}\right)^{2}\right]\right\} .
\end{gathered}
$$

In order to further demonstrate the dynamical features of MIN and GD, we specify our study to two exemplary reservoir spectra widely used in the literature, flat spectrum and Lorentzian spectrum. First, we consider the case that both spins are respectively embedded into two equal but independent flat spectral reservoirs,

$$
J_{1}(\omega)=J_{2}(\omega)=\gamma,
$$

where $\gamma$ is a constant that is commonly used as the Markov approximation with the interval of the spectral density much broader than the corresponding energy scale of the system. For this set of spectra, we obtain the time-dependent coefficients,

$$
\xi_{1}=\xi_{2}=\mathrm{e}^{-\gamma t / 2}, \chi_{1}=\chi_{2}=\left(1-\mathrm{e}^{-\gamma t}\right)^{1 / 2} .
$$

We plot the time evolution of $N$ and $D$ for different partitions as in Figure 1, where we take two initial states for spins: Bell state with $\alpha=\beta=1 / \sqrt{2}$ and Belllike state with $\alpha=1 / \sqrt{8}, \beta=\sqrt{7 / 8}$. From the figure along with the analytical expressions of Equations (12)-(15), we can find the following features. Firstly, there is clearly correlation transference between the spins and reservoirs [Figure 1(a) and 1(b)]. Both $N$ and $D$ initially stored in the spins run into reservoirs gradually. And in the equilibrium, $\lim _{t \rightarrow \infty} N\left(\rho_{r_{1} r_{2}}(t)\right)=N\left(\rho_{s_{1} s_{2}}(0)\right)$ and $\lim _{t \rightarrow \infty} D\left(\rho_{r_{1} r_{2}}(t)\right)=D\left(\rho_{s_{1} s_{2}}(0)\right)$, i.e., the transference is complete. Secondly, both $N$ and $D$ of the spins deplete gradually and no sudden death occurs in the process of transference, which is different from the concurrence [20]. Indeed, the concurrence of the spin system is $C=\max \left\{0,\left[|\alpha \beta|-|\beta|^{2}\left(1-\mathrm{e}^{-\gamma t}\right)\right] \mathrm{e}^{-\gamma t}\right\}$. When

$|\alpha|<|\beta|$ (i.e., the Bell-like initial state in the figure), it will occur sudden death. Thirdly, due to the interaction of the spins with their own reservoirs, any spin $s_{1}\left(s_{2}\right)$ becomes correlated with its reservoir $r_{1}\left(r_{2}\right)$ as shown in Figure 1(c). Also, $s_{1}$ tangles with $r_{2}$ [Figure 1(d)] due to the initial correlation of the spins. However, at the end of evolution there is no correlation between any one of the spins and reservoirs. Therefore, the spin system can finally be discarded without any effect, and all correlations transfer to the reservoirs. Lastly, we find that the
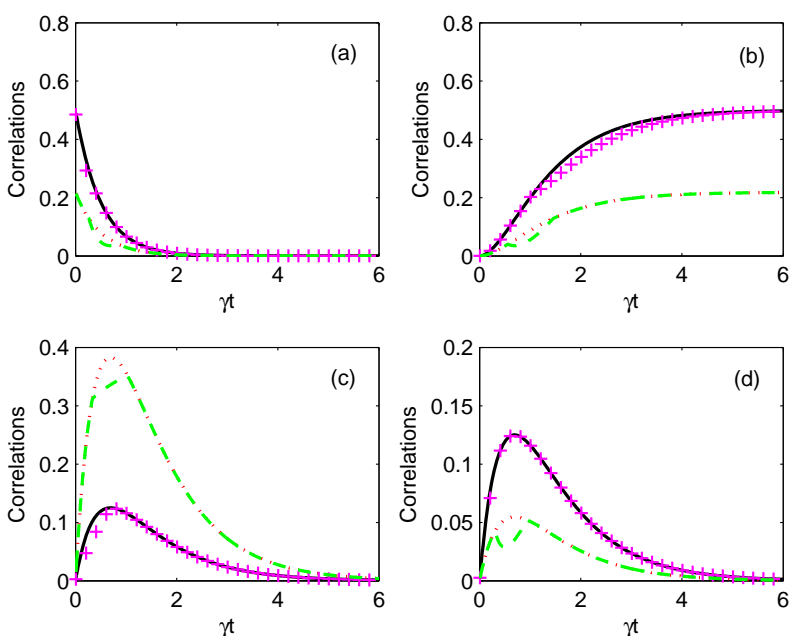

Figure 1. (Color online) Evolution of $N$ and $D$ among different partitions with flat spectral density and for the double-excitation initial spin state $|\phi(0)\rangle$. Solid black line and purple plus sign denote respectively $N$ and $D$ for Bell state. Dotted red line and dashed green line denote respectively $N$ and $D$ for Bell-like state with $\alpha=1 / \sqrt{8}, \beta=\sqrt{7 / 8}$. (a) Spins $s_{1}$ with $s_{2}$; (b) Reservoirs $r_{1}$ with $r_{2}$; (c) Spin $s_{1}$ with reservoir $r_{1}$; (d) Spin $s_{1}$ with reservoir $r_{2}$. 
time evolutions of $N$ and $D$ in this flat Markovian reservoir are roughly in accordance, only in few time intervals they behave large difference. This result highlights in some sense the relation between the two definitions. Note that there are clearly some non-smooth points (sudden changes) on the evolution of $D$ (more obvious in Figure 1(c)), which are due to the max operation in the expressions of $D$ [see Equations (13) and (15)].

Up to now, we have mainly concentrated on the case of flat reservoirs where the dynamics is Markovian. There will be different features for structured reservoirs which will lead to obvious non-Markovian dynamics. Assume that the two spins are now embedded in the local Lorentzian reservoirs with spectra,

$$
J_{i}(\omega)=\frac{W^{2} \lambda}{\pi} \frac{1}{\left(\omega_{0}-\omega-\Delta_{i}\right)^{2}+\lambda^{2}} .
$$

This spectrum is commonly used to describe a twolevel atom in an imperfect cavity. Where $\omega_{0}$ is the Bohr frequency of the spins, and $\Delta_{i}=\omega_{0}-\omega_{i c}$ is the frequency detuning between spin $i$ and its cavity mode. The quantity $\lambda$ is the photon-leakage rate of the cavity whose inverse denotes the reservoir correlation time. The ideal cavity limit is obtained for $\lambda \rightarrow 0$, where

$J(\omega)=W^{2} \delta\left(\omega-\omega_{0}\right)$, displaying a strong non-Markovian effect. For the case of resonance

$\Delta_{1}=\Delta_{2}=0$, we have,

$$
\begin{aligned}
& \xi_{1}=\xi_{2}=\mathrm{e}^{-\lambda t / 2}[\cosh (\Omega t / 2)+\lambda / \Omega \sinh (\Omega t / 2)], \\
& \chi_{1}=\chi_{2}=\sqrt{1-\xi_{1}^{2}},
\end{aligned}
$$

with $\Omega=\sqrt{\lambda^{2}-4 W^{2}}$. The time evolution of $N$ and $D$ for different partitions in this case is presented in Figure 2. For clarity, we only present the evolution of $N$ and $D$ for the initial state of the spins to be in Bell state. The distinctive property compared with the case of flat reservoirs is the oscillation in the evolution of $N$ and $D$, which is the result of non-Markovian effect. Other alike properties include: complete correlation transference between the spins and reservoirs, no sudden death of correlations for the spins in the transferring process, the vanishing correlations between spins and reservoirs at the end of evolution, and the roughly overlapping evolutional curves for $N$ and $D$.

For the case of non-resonance $\Delta_{i} \neq 0$, we obtain,

$$
\begin{aligned}
& \xi_{i}=\mathrm{e}^{-\left(\lambda-i \Delta_{i}\right) t / 2}\left[\cosh \left(\Omega_{i} t / 2\right)+\frac{\lambda-i \Delta_{i}}{\Omega_{i}} \sinh \left(\Omega_{i} t / 2\right)\right], \\
& \chi_{i}=\sqrt{1-\xi_{i}^{2}},
\end{aligned}
$$

with $\Omega_{i}=\sqrt{\left(\lambda-i \Delta_{i}\right)^{2}-4 W^{2}}$. The time evolution of $N$ and $D$ for different partitions is plotted in Figure 3, where we also choose $W=20 \lambda$ and $\Delta_{1}=\Delta_{2}=20 \lambda$. Similarly, the oscillation due to the non-Markovian effect
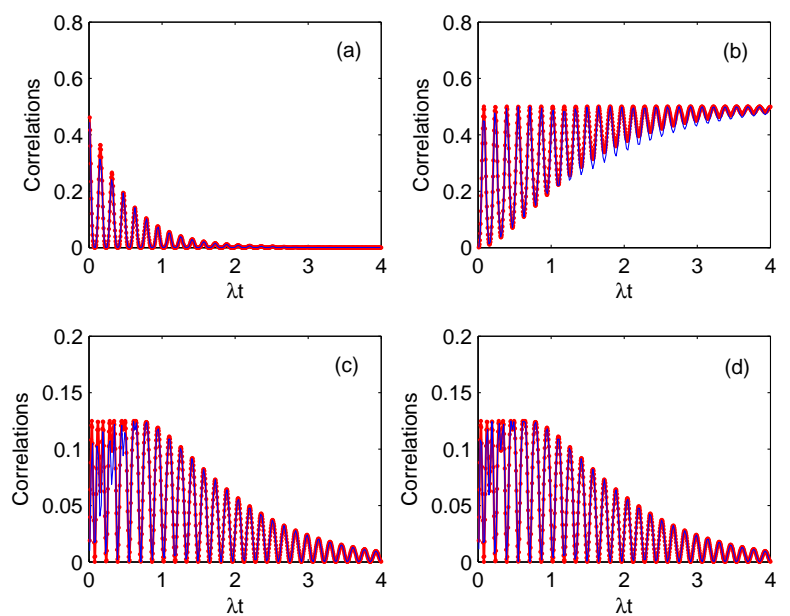

Figure 2. (Color online) Dynamics of $N$ and $D$ for the double-excitation initial Bell state of spins and in the Lorentz spectrum with $W=20 \lambda, \Delta_{1}=\Delta_{2}=0$. Red dotted and blue lines denote respectively $N$ and $D$. (a) Spins $s_{1}$ with $s_{2}$; (b) Reservoirs $r_{1}$ with $r_{2}$; (c) Spin $s_{1}$ with reservoir $r_{1}$; (d) Spin $s_{1}$ with reservoir $r_{2}$.
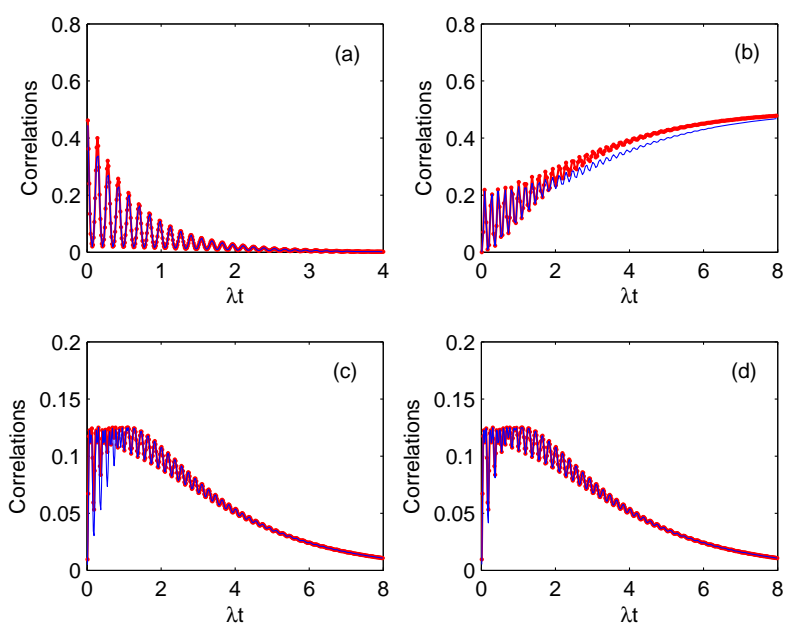

Figure 3. (Color online) Evolution of $N$ and $D$ among different partitions with non-resonant Lorentz spectrum ( $\left.W=20 \lambda, \Delta_{1}=\Delta_{2}=0\right)$ and for the double-excitation initial Bell state of spins. Red dotted and blue lines denote respectively $N$ and $D$. (a) Spins $s_{1}$ with $s_{2}$; (b) Reservoirs $r_{1}$ with $r_{2}$; (c) Spin $s_{1}$ with reservoir $r_{1}$; (d) Spin $s_{1}$ with reservoir $r_{2}$.

also exists, but the amplitude of oscillation becomes smaller compared with the resonant case. From Equations (12)-(15), we can also find that the correlation transference between the spins and reservoirs is also complete, but the transferring time becomes longer compared with the corresponding resonant case. This is because the non-resonant effect decreases the coupling between the spin and its reservoir. The steady values of $N$ 
and $D$ for $\rho_{s_{1} r_{1}}$ and $\rho_{s_{1} r_{2}}$ are also zero [not show completely in Figure 3(c) and (d)]. Note that the evolutions of $N$ and $D$ also roughly coincide.

\subsection{Single-Excitation Dynamics of MIN and GD}

We now study the correlation evolution for another type of initial state which has only one excitation in the spin system,

$$
|\psi(0)\rangle=\left(\alpha|0\rangle_{s_{1}}|1\rangle_{s_{2}}+\beta|1\rangle_{s_{1}}|0\rangle_{s_{2}}\right)|0\rangle_{r_{1}}|0\rangle_{r_{2}},
$$

with $|\alpha|^{2}+|\beta|^{2}=1$. The dynamical evolution of the overall system in this case can be solved as,

$$
\begin{aligned}
& |\psi(t)\rangle \\
& =\alpha\left(\xi_{2}(t)|0\rangle_{s_{1}}|1\rangle_{s_{2}}|0\rangle_{r_{1}}|0\rangle_{r_{2}}+\chi_{2}(t)|0\rangle_{s_{1}}|0\rangle_{s_{2}}|0\rangle_{r_{1}}|1\rangle_{r_{2}}\right) \\
& \quad+\beta\left(\xi_{1}(t)|1\rangle_{s_{1}}|0\rangle_{s_{2}}|0\rangle_{r_{1}}|0\rangle_{r_{2}}+\chi_{1}(t)|0\rangle_{s_{1}}|0\rangle_{s_{2}}|1\rangle_{r_{1}}|0\rangle_{r_{2}}\right),
\end{aligned}
$$

here $\xi_{i}(t)$ and $\chi_{i}(t)$ are also determined by Equations (9) and (10). $N$ and $D$ for different partitions are,

$$
N\left(\rho_{s_{1} s_{2}}\right)=2\left|\alpha \beta \xi_{1} \xi_{2}\right|^{2},
$$

$$
\begin{aligned}
& D\left(\rho_{s_{1} s_{2}}\right) \\
= & \frac{1}{4}\left\{\left(1-2\left|\beta \xi_{1}\right|^{2}\right)^{2}+8\left|\alpha \beta \xi_{1} \xi_{2}\right|^{2}+\left(1-2\left|\alpha \xi_{2}\right|^{2}-2\left|\beta \xi_{1}\right|^{2}\right)^{2}\right. \\
& -\max \left[4\left|\alpha \beta \xi_{1} \xi_{2}\right|^{2},\left(1-2\left|\beta \xi_{1}\right|^{2}\right)^{2}+\left(1-2\left|\alpha \xi_{2}\right|^{2}-2\left|\beta \xi_{1}\right|^{2}\right)^{2}\right],
\end{aligned}
$$

and

$$
N\left(\rho_{r_{1} r_{2}}\right)=2\left|\alpha \beta \chi_{1} \chi_{2}\right|^{2}
$$

$$
\begin{aligned}
& D\left(\rho_{r_{1} r_{2}}\right) \\
= & \frac{1}{4}\left\{\left(1-2\left|\beta \chi_{1}\right|^{2}\right)^{2}+8\left|\alpha \beta \chi_{1} \chi_{2}\right|^{2}+\left(1-2\left|\alpha \chi_{2}\right|^{2}-2\left|\beta \chi_{1}\right|^{2}\right)^{2}\right. \\
& \left.-\max \left[4\left|\alpha \beta \chi_{1} \chi_{2}\right|^{2},\left(1-2\left|\beta \chi_{1}\right|^{2}\right)^{2}+\left(1-2\left|\alpha \chi_{2}\right|^{2}-2\left|\beta \chi_{1}\right|^{2}\right)^{2}\right]\right\} .
\end{aligned}
$$

It is interesting to find that $N\left(\rho_{s_{1} s_{2}}\right)$ and $N\left(\rho_{r_{1} r_{2}}\right)$ have the same expressions for one- and two-excitation spin states [compare Equations (23) and (25) with Equations (12) and (14)]. So do $N\left(\rho_{s_{1} r_{1}}\right)$ and $N\left(\rho_{s_{1} r_{2}}\right)$ [not given in the text]. While for $D$, there is not similar result.

When the two spins coupled to flat reservoirs, the parameters $\xi_{i}$ and $\chi_{i}$ are still given by Equation (17). We show the correlation transference of this case in Figure 4, which has roughly the similar properties as the corresponding two-excitation case.

If the spins couple to resonant and non-resonant Lorentz reservoirs, the corresponding parameters $\xi_{i}$ and $\chi_{i}$ are given respectively by Equations (19) and (20). The corresponding correlation transference depicted in Figures $\mathbf{5}$ and $\mathbf{6}$ are still similar to that of two-excitation case.
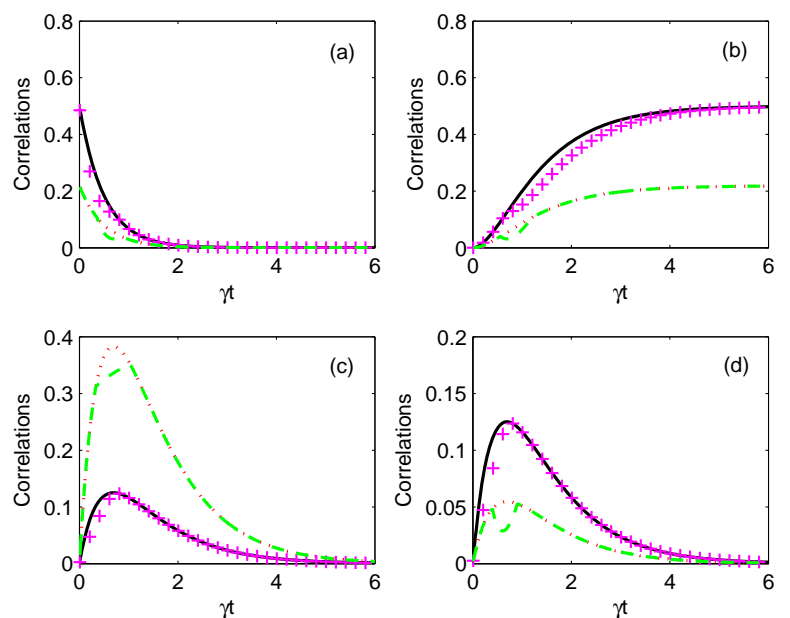

Figure 4. (Color online) Dynamics of $N$ and $D$ for the single-excitation initial spin state $|\psi(0)\rangle$ and flat spectral density. Solid black line and purple plus sign denote respectively $N$ and $D$ for initial Bell state, while dotted red line and dashed green line for initial Bell-like state with $\alpha=1 / \sqrt{8}, \beta=\sqrt{7 / 8}$. (a) Spins $s_{1}$ with $s_{2}$; (b) Reservoirs $r_{1}$ with $r_{2}$; (c) Spin $s_{1}$ with reservoir $r_{1}$; (d) Spin $s_{1}$ with reservoir $r_{2}$.
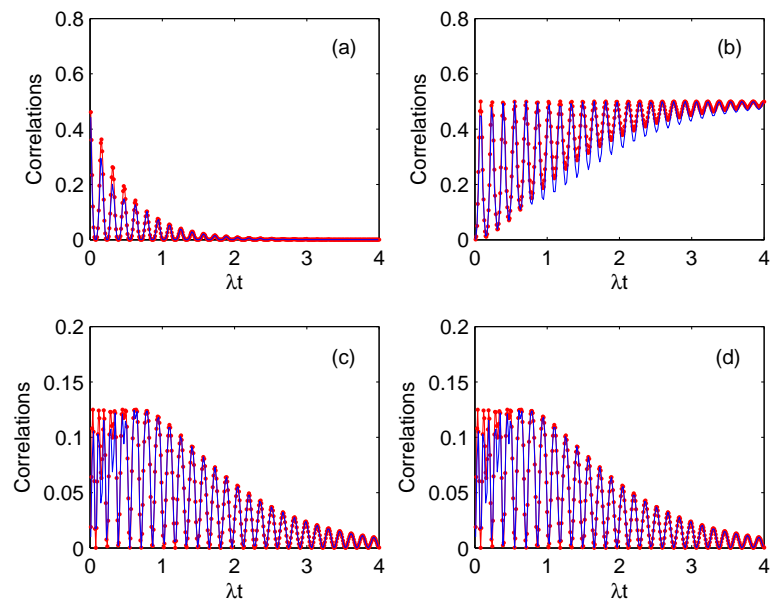

Figure 5. (Color online) Dynamics of $N$ and $D$ for the single-excitation initial Bell state of spins and for resonant Lorentz spectra $\left(W=20 \lambda, \Delta_{1}=\Delta_{2}=0\right)$. Red dotted and blue lines denote respectively $N$ and $D$. (a) Spins $s_{1}$ with $s_{2}$; (b) Reservoirs $r_{1}$ with $r_{2}$; (c) Spin $s_{1}$ with reservoir $r_{1}$; (d) Spin $s_{1}$ with reservoir $r_{2}$. 

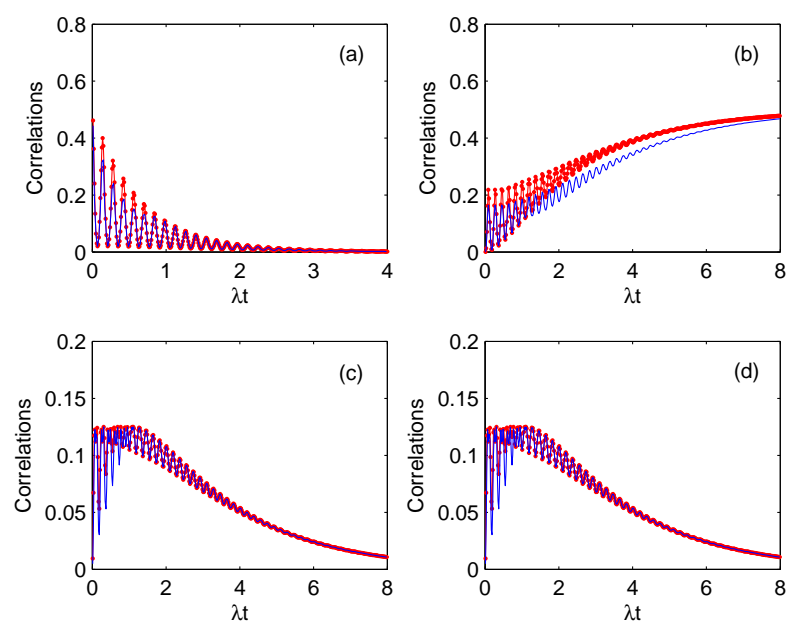

Figure 6. (Color online) Evolution of $N$ and $D$ among different partitions for non-resonant Lorentz spectrum $\left(W=20 \lambda, \Delta_{1}=\Delta_{2}=20 \lambda\right)$ and for the single-excitation initial

Bell state of spins. Red dotted and blue lines denote respectively $N$ and $D$. (a) $s_{1}$ with $s_{2}$; (b) $r_{1}$ with $r_{2}$; (c) $s_{1}$ with $r_{1}$; (d) $s_{1}$ with $r_{2}$.

\section{Quant Beat for MIN and GD}

Quantum beat is a very interesting phenomenon in quantum optics. We discussed the entanglement and discord quantum beats in detail early [31] in open two-level systems. Here, we find that for the time evolution of $N$ and $D$ a similar phenomenon also appears. Let us still assume that the two spins are plugged into their own Lorentz reservoirs with spectral density given by Equation (18). For simplicity, we only discuss the non-resonant and two-excitation case. For resonant or/and oneexcitation case, similar phenomenon of quantum beat for $N$ and $D$ also exists. The quantum correlations $N$ and $D$ for the spins and the reservoirs are described respectively by Equations (12)-(15) with parameters $\xi_{i}$ and $\chi_{i}$ given by Equation (20). The corresponding time evolutions are depicted in Figure 7, where

$\alpha=\beta=1 / \sqrt{2}$ and $\Delta_{1}=50 \lambda, \Delta_{2}=45 \lambda$. The tiny difference between $\Delta_{1}$ and $\Delta_{2}$ is the demand of observing quantum beat. Only in this way can we induce two harmonic oscillations with tiny different frequencies, whose interfering superposition forms quantum beat. These quantum beats originate from non-Markovian effect, as no any direct or mediated interaction exists between the two spins or the two reservoirs. It is the result of both non-Markovian effect and quantum interference. The detailed mathematical analysis may be consulted in Reference [31].

\section{Conclusions}

We have studied the dynamics of both $N$ and $D$ in the spin-boson model, where two independent spins re-
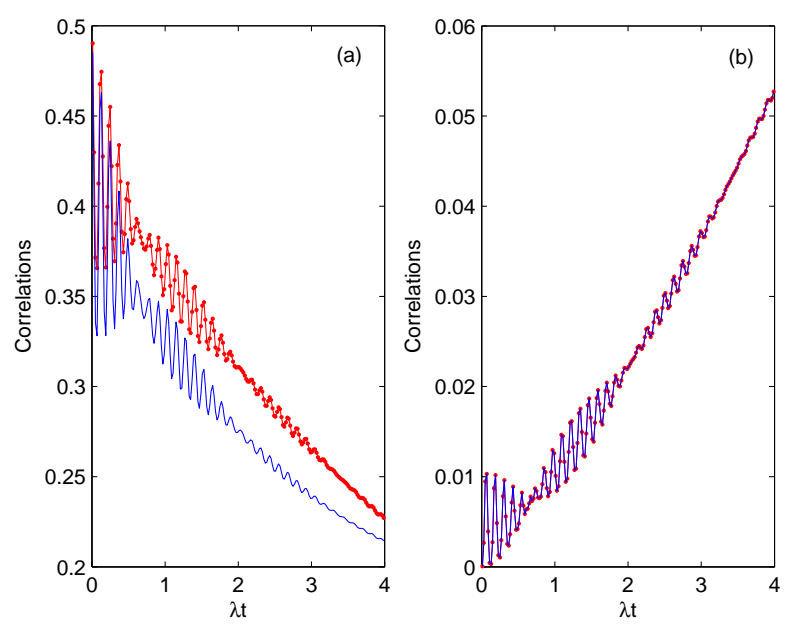

Figure 7. (Color online) Dynamics for $N$ and $D$ for the double-excitation initial Bell state of spins and for nonresonant Lorentz spectrum

$\left(W=10 \lambda, \Delta_{1}=50 \lambda, \Delta_{2}=45 \lambda\right)$. (a) $s_{1}$ with $s_{2}$. (b) $r_{1}$ with $r_{2}$. Red dotted and blue lines denote respectively $N$ and $D$.

spectively couple to their local reservoirs. We considered two large types of initial states of the spins, i.e., one- and two-excitation states. We found that $N$ of different partitions is identical for the two types of initial states, while $D$ depends on the initial states. In the situations of flat and Lorentz spectral reservoirs, we have both analytically and numerically simulated the time evolutions of $N$ and $D$. We found that there exist complete correlation transference between the system and reservoirs, all $N$ and $D$ initially stored in the spins completely transfer to reservoirs at the end of evolution. There is no sudden death of correlations for the spin system in the transferring process, which forms bright contrast to the quantum entanglement. For flat spectral reservoirs, there is no oscillation for $N$ and $D$ in the transferring process. While for memory Lorentz reservoirs, oscillation appears which is the symbol of non-Markovian effect. In particular, when the detunings of the spins with their reservoirs have tiny difference, the quantum beats for $N$ and $D$ are observed which signify the quantum interference between the two dynamics of the spins through quantum correlations. For the Lorentz reservoirs and in the case of resonance between the spins and their reservoirs, the oscillation amplitudes of $N$ and $D$ are larger, and the time of the correlation transference is shorter. While in the detunings (particularly for large detunings), the oscillation amplitudes become smaller and the transferring time becomes longer. Finally, we found that though the different definitions, the evolutions of $N$ and $D$ are very close for most cases under consideration. This is an astonishing result which highlights in some sense the relation between the two definitions. Also note that it may happen sudden change for the 
evolution of $D$.

Note that a similar work for the evolutions of the quantum and classical correlations was done [29]. The transference of $N$ and $D$ studied here has some similar properties to that of quantum and classical correlations. However it also appears many differences, for example, $N$ is the same for one- and two-excitation cases, and $D$ may happen sudden change in the evolutional process. Especially, $N$ and $D$ have very coincident evolutions in most cases under considerations.

Quantum correlation is a kind of unique characteristics of quantum system which could be a new resource in quantum information technology. Exploring the relation between various quantum correlations and finding the dynamical rules of them in practical environments can not only contribute to a better understanding of the concepts, but also offer possible references for applications.

\section{Acknowledgements}

This work is supported by the National Natural Science Foundation of China (Grant Nos. 11275064, 11075050), Specialized Research Fund for the Doctoral Program of Higher Education (Grant No. 20124306110003) the Construct Program of the National Key Discipline, the Program for Changjiang Scholars and Innovative Research Team in University under Grant No. IRT0964, and Hunan Provincial Natural Science Foundation under Grant No. 11JJ7001.

\section{REFERENCES}

[1] S. Luo, "Using Measurement-Induced Disturbance to Characterize Correlations as Classical or Quantum," Physical Review A, Vol. 77, No. 2, 2008, Article ID: 022301. doi:10.1103/PhysRevA.77.022301

[2] M. A. Nielsen and I. L. Chuang, "Quantum Computation and Quantum Information," Cambridge University Press, Cambridge, 2000.

[3] H. Ollivier and W. H. Zurek, "Quantum Discord: A Measure of the Quantumness of Correlations," Physical Review Letters, Vol. 88, No. 1, 2001, Article ID: 017901. doi:10.1103/PhysRevLett.88.017901

[4] A. Datta, A. Shaji and C. M. Caves, "Quantum Discord and the Power of One Qubit," Physical Review Letters, Vol. 100, No. 5, 2008, Article ID: 050502. doi:10.1103/PhysRevLett.100.050502

[5] B. P. Lanyon, M. Barbieri, M. P. Almeida and A. G. White, "Experimental Quantum Computing without Entanglement," Physical Review Letters, Vol. 101, No. 20, 2008, Article ID: 200501. doi:10.1103/PhysRevLett.101.200501

[6] R. Dillenschneider, "Quantum Discord and Quantum Phase Transition in Spin Chains," Physical Review B, Vol. 78, No. 22, 2008, Article ID: 224413. doi:10.1103/PhysRevB.78.224413
[7] M. S. Sarandy, "Classical Correlation and Quantum Discord in Critical Systems," Physical Review A, Vol. 80, No. 2, 2009, Article ID: 022108. doi:10.1103/PhysRevA.80.022108

[8] J. Cui and H. Fan, "Correlations in the Grover Search," Journal of Physics A: Mathematical and Theoretical, Vol. 43, No. 4, 2010, Article ID: 045305. doi:10.1088/1751-8113/43/4/045305

[9] S. Luo, "Quantum Discord for Two-Qubit Systems," Physical Review A, Vol. 77, No. 4, 2008, Article ID: 042303. doi:10.1103/PhysRevA.77.042303

[10] G. Adesso and A. Datta, "Quantum versus Classical Correlations in Gaussian States," Physical Review Letters, Vol. 105, No. 3, 2010, Article ID: 030501. doi:10.1103/PhysRevLett.105.030501

[11] P. Giorda and M. G. A. Paris, "Gaussian Quantum Discord," Physical Review Letters, Vol. 105, No. 2, 2010, Article ID: 020503. doi:10.1103/PhysRevLett.105.020503

[12] B. Dakic, V. Vedral and C. Brukner, "Necessary and Sufficient Condition for Nonzero Quantum Discord," Physical Review Letters, Vol. 105, No. 19, 2010, Article ID: 190502. doi:10.1103/PhysRevLett.105.190502

[13] S. Luo and S. Fu, "Measurement-Induced Nonlocality," Physical Review Letters, Vol. 106, No. 12, 2011, Article ID: 120401. doi:10.1103/PhysRevLett.106.120401

[14] S. Luo and S. Fu, "Geometric Measure of Quantum Discord," Physical Review A, Vol. 82, No. 3, 2010, Article ID: 034302. doi:10.1103/PhysRevA.82.034302

[15] S. Luo and S. Fu, "Evaluating the Geometric Measure of Quantum Discord," Theoretical and Mathematical Physics, Vol. 171, No. 3, 2012, pp. 870-878. doi:10.1007/s11232-012-0082-x

[16] B. Bellomo, R. Lo Franco and G. Compagno, "Dynamics of Geometric and Entropic Quantifiers of Correlations in Open Quantum Systems," Physical Review A, Vol. 86, No. 1, 2012, Article ID: 012312. doi:10.1103/PhysRevA.86.012312

[17] B. Bellomo, G. L. Giorgi, F. Galve, R. Lo Franco, G. Compagno and R. Zambrini, "Unified View of Correlations Using the Square Norm Distance," Physical Review A, Vol. 85, No. 3, 2012, Article ID: 032104. doi:10.1103/PhysRevA.85.032104

[18] K. Ann and G. Jaeger, "Finite-Time Destruction of Entanglement and Non-Locality by Environmental Influences," Foundations of Physics, Vol. 39, No. 7, 2009, pp. 790-828. doi:10.1007/s10701-009-9295-8

[19] B. Bellomo, G. Compagno, R. Lo Franco, A. Ridolfo and S. Savasta, "Dynamics and Extraction of Quantum Discord in Multipartite Open Systems," International Journal of Quantum Information, Vol. 9, No. 7-8, 2011, pp. 1665-1676.

[20] T. Yu and J. H. Eberly, "Finite-Time Disentanglement via Spontaneous Emission," Physical Review Letters, Vol. 93, No. 14, 2004, Article ID: 140404. doi:10.1103/PhysRevLett.93.140404

[21] B. Bellomo, G. Compagno, A. D’Arrigo, G. Falci, R. Lo Franco and E. Paladino, "Entanglement Degradation in the 
Solid State: Interplay of Adiabatic and Quantum Noise," Physical Review A, Vol. 81, No. 6, 2010, Article ID: 062309. doi:10.1103/PhysRevA.81.062309

[22] T. Werlang, S. Souza, F. F. Fanchini and C. J. Villas Boas, "Robustness of Quantum Discord to Sudden Death," Physical Review A, Vol. 80, No. 2, 2009, Article ID: 024103. doi:10.1103/PhysRevA.80.024103

[23] B. Bellomo, R. Lo Franco and G. Compagno, "Non-Markovian Effects on the Dynamics of Entanglement," Physical Review Letters, Vol. 99, No. 16, 2007, Article ID: 160502. doi:10.1103/PhysRevLett.99.160502

[24] R. Lo Franco, B. Bellomo, S. Maniscalco and G. Compagno, "Dynamics of Quantum Correlations in Two-Qubit Systems within Non-Markovian Environments," International Journal of Modern Physics B, Vol. 27, No. 1-3, 2013, Article ID: 1345053.

[25] R. Lo Franco, A. D’Arrigo, G. Falci, G. Compagno and E. Paladino, "Entanglement Dynamics in Superconducting Qubits Affected by Local Bistable Impurities," Physica Scripta, Vol. T147, 2012, Article ID: 014019. doi:10.1088/0031-8949/2012/T147/014019

[26] B. Bellomo, G. Compagno, R. Lo Franco, A. Ridolfo and S. Savasta, "Entanglement Dynamics of Two Independent Cavity-Embedded Quantum Dots," Physica Scripta, Vol. T143, 2011, Article ID: 014004. doi:10.1088/0031-8949/2011/T143/014004
[27] R. Lo Franco, B. Bellomo, E. Andersson and G. Compagno, "Revival of Quantum Correlations without System-Environment Back-Action," Physical Review A, Vol. 85, No. 3, 2012, Article ID: 032318. doi:10.1103/PhysRevA.85.032318

[28] Q. J. Tong, J. H. An, H. G. Luo and C. H. Oh, "Mechanism of Entanglement Preservation," Physical Review A, Vol. 81, No. 5, 2010, Article ID: 052330. doi:10.1103/PhysRevA.81.052330

[29] R. C. Ge, M. Gong, C. F. Li, J. S. Xu and G. C. Guo, "Quantum Correlation and Classical Correlation Dynamics in the Spin-Boson Model," Physical Review A, Vol. 81, No. 6, 2010, Article ID: 064103. doi:10.1103/PhysRevA.81.064103

[30] B. Bellomo, R. Lo Franco, S. Maniscalco and G. Compagno, "Entanglement Trapping in Structured Environments," Physical Review A, Vol. 78, No. 6, 2008, Article ID: 060302(R). doi:10.1103/PhysRevA.78.060302

[31] H. S. Zeng, Y. P. Zheng, N. Tang and G. Y. Wang, "Correlation Quantum Beats Induced by Non-Markovian Effect," Quantum Information Process, Vol. 12, No. 4, 2013, pp. 1637-1650. doi:10.1007/s11128-012-0437-0

[32] C. E. Lopez, G. Romero and J. C. Retamal, "Dynamics of Entanglement Transfer through Multipartite Dissipative Systems," Physical Review A, Vol. 81, No. 6, 2010, Article ID: 062114. doi:10.1103/PhysRevA.81.062114 